Molecular

\section{genetics handbook}

Handbook of Genetics. Vol. 5: Molecular Genetics. Edited by R. C. King. Pp.xi+667. (Plenum: New York and London, 1976.) \$51.

THE fifth volume of the Handbook of Genetics in many ways belies its title. The book is in four sections devoted to the molecular organisation of chromosomes (six chapters), gene transcripts (five chapters), chloroplasts and mitochondria (five chapters), and a single chapter in a section called mutant enzymes. Only the chapters in the section on chloroplasts and mitochondria seem truly to combine both a molecular and genetical approach and deserve the title molecular genetics.

Particularly disappointing is the pot pourri section on molecular organisation of chromsomes, which manages to present a somewhat incoherent treatment of the subject in spite of excellent reviews of chromosomal protein structure and function, and of organisation and size of replicons. Individual chapters on repeated DNA in eukaryotes, cytological localisation of repeated DNAs, chromosomal proteins, organisation of genetic material in the macronucleus of hypotrichous ciliates, histones of sperm, and organisation and size of replicons, do not easily relate to each other and the section as a whole presents no overall picture of chromosome organisation. The chapter on repeated DNA in eukaryotes, by limiting itself to molecular renaturation studies, manages to ignore much interesting and relevant data provided by sequencing, restriction site mapping and electron microscopy of repeated DNAs.

The chapter on chromosomal proteins, although admirable in many ways, contains no reference to the unit of chromatin (nucleosome) and models which have been proposed for its structure. Such an omission can hardly be of the authors own making since it seems evident from the reference list that this chapter was complete by the end of 1973 (the only 1974 paper quoted is by the authors themselves) and the same seems to hold for the majority of chapters in this section; one exception being the chapter on macronuclear DNA of hypotrichs, which has references to 1976. Judging by the latest references in chapters of other sections they were completed towards the end of 1974 or beginning of 1975, although the chapter on mutant enzymes has no references after 1972 . It is a pity that the relatively long lag between writing and publishing the chapters in section one has reduced its effectiveness as a contemporary review of a rapidly changing area of investigation.

The other sections are more successful. Section two, gene transcripts, has chapters on nuclear RNA, RNA transcription and ribosomal protein assembly in Drosophila melanogaster, cytoplasmic mRNA, tRNAs, and eukaryotic ribosomes; whereas section three, chloroplasts and mitochondria, has chapters on chloroplast DNA (physical and genetic studies), chloroplast ribosomes, nucleocytoplasmic interaction in Acetabularia, mitochondrial DNA, and mitochondrial ribosomes. The chapters on cytological localisation of repeated DNAs, histones of sperm, transfer RNAs, and mutant enzymes are little more than exhaustive catalogues of the structure, location or primary sequence of particular macromolecules.

\section{Chemical relaxation}

Relaxation Kinetics. By C. F. Bernasconi. Pp. xi+288. (Academic: New York and London, 1976.) \$29.50; $£ 20.95$.

THERE can be no getting away from it -a large proportion of chemical reactions are fast. Generations of students may have obtained their grounding in chemical kinetics with reactions whose half-lives lie conveniently in the time range of minutes or hours, but the fact is that such reactions are the exception rather than the rule. One has only to recall the particular premium placed on the time factor in many biologically and industrially important reactions to appreciate the significance of our being able to measure the kinetics of rapid reactions.

It is not possible to apply classical kinetic techniques to many of these reactions but over the past fifteen years or so a variety of methods have been developed which have made most of them susceptible to study. All of these methods involve electronic equipment and the prospect of having to build one's own apparatus, together with the fact that a rather different approach is generally required in the analysis of the data, has tended to scare away all but the most physically-minded chemists. Times are, however, changing and it is thanks to the increasing availability of suitable commercial equipment and monographs such as Dr Bernasconi's that more and more chemists are reporting rate constants and
In spite of some omissions which might have been included in a book with this title-for instance, considerable space is devoted to histones but no mention is made of histone genes, and similarly there is no mention of tRNA genes although all the known tRNA sequences are tabulated-there is a great deal of worthwhile and well presented material. As a reference book in certain areas, it should prove valuable.

The book has an author and subject index. But the subject index has innumerable deficiencies, and I pick one example to illustrate this point. The only reference to "globin" contains an interesting cross-reference "see Immunoglobin". There is, however, no entry under "Immunoglobin"; but under "Immunoglobulin" there is a reference to "chain of rabbit reticulocyte"! Peter Ford

Peter Ford is Lecturer in the Department of Molecular Biology, University of Edinburgh, UK.

activation parameters where in the past they would have used some such phrase as "too fast to measure".

The first two-thirds of Dr Bernasconi's book deals with the general theory of chemical relaxation and is largely given over to the problem of relating the relaxation time (or times) and the rate constants for different mechanistic schemes. Also included are a couple of chapters (out of ten) on the topic of relaxation amplitudes. The remainder of the book is concerned with the various experimental methods.

The author says that he has attempted to write a comprehensive treatment of the subject in the language of the organic or physical-organic chemist and in this I think he has on the whole been successful. Certainly, the mathematics should not overtax any mechanistically-minded research chemist (contrary to the impression he might gain on casually leafing through it) and there are many useful hints and practical comments hidden (the correct word, unfortunately) throughout the book.

Where I would be a little less than fulsome in praising the book is for its comprehensiveness. Although his choice will certainly not be universally applauded, it seems to me that Dr Bernasconi has quite properly and freely exercised his author's discretion on the topics for inclusion. Presumably, this is the reason behind his implication (with which I would not agree) that this is not a book for the physical-inorganic chemist.

D. N. Hague

D. N. Hague is Senior Lecturer in Chemistry, University of Kent at Canterbury, UK. 